\title{
Peacocke on magnitudes and numbers
}

\author{
Øystein Linnebo'
}

Accepted: 26 April 2020/Published online: 27 September 2020

(C) The Author(s) 2020

\begin{abstract}
Peacocke's recent The Primacy of Metaphysics covers a wide range of topics. This critical discussion focuses on the book's novel account of extensive magnitudes and numbers. First, I further develop and defend Peacocke's argument against nominalistic approaches to magnitudes and numbers. Then, I argue that his view is more Aristotelian than Platonist because reified magnitudes and numbers are accounted for via corresponding properties and these properties' application conditions, and because the mentioned objects have a "shallow nature" relative to the corresponding properties. The result is an asymmetric conception of abstraction, which contrasts with the neo-Fregeans' but has important tenets in common with an approach that I have recently developed.
\end{abstract}

Keywords Extensive magnitudes · Numbers · Anti-nominalism · Aristotelianism · Frege $\cdot$ Abstraction · Reification · Paradox

\section{Introduction}

The Primacy of Metaphysics (Peacocke 2019) is about the relation between representation and reality, in particular about their relative explanatory priority. Although this is "a timeless, $u r$-issue in philosophy" (p. 10), the book develops a distinctive and novel position, which is often plausible and always interesting. As one might expect, given the topic and the author, the book covers a lot of ground: from magnitudes and numbers, through space and time, to the self, as well as our language and thought about each of these domains. I will here focus on magnitudes and number, the discussion of which comprises roughly a third of the book, where Christopher Peacocke (henceforth CP) makes a number of important contributions.

\footnotetext{
Øystein Linnebo

oystein.linnebo@ifikk.uio.no

1 University of Oslo, Oslo, Norway
} 
First, however, I wish to make some remarks about the project as a whole. According to meaning-first views, theories of meaning and intentional content concerning a domain are always explanatory prior to the metaphysics of the domain. This approach has been pioneered by Michael Dummett and sympathetically discussed by Robert Brandom and Crispin Wright—with Kant lurking in the shadows. Although Peacocke's framing of the discussion has a clear and acknowledged debt to Dummett, he strongly rejects the latter's meaning-first view. Instead, CP defends:

\section{Primary Thesis}

The metaphysics of a domain is involved in the philosophical explanation of the nature of the meanings of sentences about that domain; and the metaphysics of a domain is involved in the philosophical explanation of the nature of intentional contents (ways of representing) concerning that domain. (p. 4)

Instead of meaning-first, we are thus given a choice between metaphysics-first (which speaks for itself) and no-priority (which holds that the metaphysics of a domain and our representation of it are explanatorily on a par). According to $\mathrm{CP}$, each of these two choices has important implications.

Let's take a closer look at how CP understands the two relata, namely 'the metaphysics of a domain' and our representation of this domain. First, what is 'the metaphysics of a domain'? CP writes:

By 'the metaphysics of a domain' I mean a theory that states truly what is constitutive of the objects, properties, and relations of that domain - a theory of what makes them the objects, properties, and relations they are. (p. 16)

Notice the centrality of questions of individuation to this conception of metaphysics. We are inquiring into what is 'constitutive' of various entities, which is glossed as a question of what "makes these entities the entities they are". This is a purely metaphysical notion of individuation, which is distinct from some semantic or metasemantic notions that are also prominent in the literature.

Concerning the second relatum, $\mathrm{CP}$ writes that we want

an explanation that does not merely specify the meanings of expressions in the relevant language, but rather a theory that says, substantively, what it is to understand those expressions. (p. 17)

That is, we want not only a semantics for the relevant language but also a metasemantic account, which explains what endows linguistic expressions and mental representations with their semantic values and, relatedly, what it is to understand an expression or have a representation. Here we are concerned with a metasemantic notion of individuation: what is it to pick out, or refer to, a particular entity in language or thought?

CP's Primary Thesis is thus at heart a thesis about the relation between two notions of individuation. The thesis states that the metaphysical notion is prior to, or on a par with, the metasemantic one. Or, with CP's Sartre-inspired slogan: individuation is prior to representation.

Why accept the Primary Thesis? CP's principal argument is fairly straightforward. 
Which relations a thinker can stand into an entity depends on the correct metaphysics of that entity. It follows that the metaphysics of a domain constrains the theory of concepts of entities of that domain. (p. 27)

I find this argument quite compelling. So as far as I am concerned, the more pressing question concerns the choice between metaphysics-first and no-priority. While CP tends to favor the former, I see a more extensive role for the latter, for reasons I explain towards the end of this note.

\section{Towards a metaphysics of extensive magnitudes}

The book mounts an impressive defense of the philosophical importance of extensive magnitudes, defined as magnitudes for which there is a natural operation of addition. Examples include lengths, durations, and masses. This contrasts with temperature or a material's hardness, which are classified as intensive magnitudes.

Chapter 2 distinguishes three notions of extensive magnitude:

(i) Magnitude types, e.g. length, duration, mass

(ii) Magnitudes themselves, e.g. length $1 \mathrm{~m}$, duration $1 \mathrm{~s}$, mass $1 \mathrm{~kg}$

(iii) Magnitude tropes, e.g. the length of this stick

As I expect CP would agree, two further notions are important as well:

(iv) Quantities, defined as the entities that have magnitudes, e.g. this ruler, this process, this bronze weight

(v) The property of having a certain magnitude, e.g. being $1 \mathrm{~m}$ long, lasting $1 \mathrm{~s}$, having mass $1 \mathrm{~kg}$.

What is the relation between all these notions? In particular, can some of them be reduced to, or eliminated in favor of, the others?

Following the ancient debate about the status of univerals, we can distinguish three broad orientations towards the metaphysics of magnitudes. At one extreme, we find nominalism, which holds that there are just concrete objects and thus no magnitudes or magnitude properties. At the opposite extreme, we find Platonism, which affirms the existence of the mentioned entities and insists that these can be made sense of regardless of how things stand with the concrete world. Somewhere in between these two extremes we find the Aristotelian view that magnitude properties (and perhaps also magnitudes) exist provided that the property is instantiated, or at least possibly instantiated. Moreover, an Aristotelian insists that in order to make sense of, or individuate, these entities, it is necessary to appeal to concrete instantiations of this magnitude, or at least the possibility of such instantiations.

Where does CP belong on this rough map of the metaphysical terrain? He is certainly no nominalist. Among the forms of realism that remain, I will argue that he is more of an Aristotelian than a Platonist. ${ }^{1}$

\footnotetext{
1 See Hossack (2020) for a recent study of numbers and other magnitudes, in many respects similar to CP's but more self-consciously Aristotelian.
} 
There is a further distinction too within the family of realist conceptions of magnitudes. I have in mind the distinction between magnitudes themselves, i.e. notion (ii), and the corresponding magnitude properties, i.e. notion (v). CP regards magnitudes as objects, in the sense that they are "entities referred to by singular terms" (p. 69). Magnitude properties, by contrast, are most naturally understood as the semantic values of predicates. What, then, is the relation between a magnitude $m$ and the property $P_{m}$ of having that magnitude? The following principle, which I call the Reification Link, plays a central role in CP's account:

$$
\square \forall x\left(\operatorname{Has}(x, m) \leftrightarrow P_{m}(x)\right)
$$

Of course, this principle forms only the beginning of an answer to our question of the relation between a magnitude proper and the corresponding magnitude property-of which more shortly.

\section{The refutation of nominalism?}

Before trying to determine the correct metaphysics of magnitudes, it is useful to review some basic measurement theory. Let us follow CP and consider Patrick Suppes' influential account of extensional quantities. ${ }^{2}$

We begin with the language. First, there is a primitive predicate ' use to express that one object is no more massive, or long, or whatever, than another. Thus, ' $x \preceq y$ ' means that the quantity $x$ is less than or equal to $y$ in the relevant respect. Next, we define a predicate ' $x \sim y$ ' as ' $x \preceq y \wedge y \preceq x$ '. There is also a primitive summation operation $\oplus$.

Finally, we define $n \cdot x$ as $\underbrace{x \oplus x \oplus \ldots \oplus x}_{\text {ntimes }}$.

Suppes' theory of extensive quantities has the following axioms:

(1) $\preceq$ is transitive

(2) $\quad(x \oplus y) \oplus z \preceq x \oplus(y \oplus z)$

(3) $x \preceq y \rightarrow x \oplus z \preceq y \oplus z$

(4) $x \npreceq y \rightarrow \exists z(x \sim y \oplus z)$

(5) $x \oplus y \npreceq x$

(6) $x \preceq y \rightarrow \exists n(y \preceq n \cdot x)$

Notice that, when this theory holds for certain quantities, it also holds for the corresponding magnitudes. In fact, this is CP's preferred interpretation. ${ }^{3}$

This theory has a number of philosophically important theorems. Let me mention one now and another in the next section. The first theorem states that $\sim$ is an equivalence relation. The question of nominalism about magnitudes can thus be put more sharply: what is the relation between a magnitude and the

\footnotetext{
2 See Suppes (1951). I have changed the notation for greater readability.

${ }^{3}$ See Peacocke (2015, Sect. 1). On this interpretation, we avoid the pesky question of interpreting sums of the form $x \oplus x$. After all, what is it to add one quantity to itself? Presumably $x \oplus x$ should be understood as $x \oplus y$, where $x \sim y$, and with $y$ "generalized out".
} 
corresponding $\sim$-equivalence class, which the nominalist can construe as just a plurality of concrete objects? ${ }^{4}$ For example, what is the relation between the magnitude $1 \mathrm{~m}$ and the class of objects of that length? Can we, as the nominalist proposes, eliminate magnitudes and magnitude properties in favor of just the objects which, loosely speaking, have this magnitude? That is, can we dispense with notions (ii) and (v) in favor of just notion (iv)?

$\mathrm{CP}$ develops an interesting argument against any such elimination or reduction, inspired by (Putnam 1969). The argument turns on the modal profile of the magnitude (or magnitude property) and the class of entities that have this magnitude. Let $a$ be a rod of length $1 \mathrm{~m}$. It is merely contingent that $a$ has this magnitude (or magnitude property). Next, let $C$ be the class of objects of length $1 \mathrm{~m}$-or perhaps, for reasons of nominalistic hygiene, the plurality of such objects. Then it is necessary that $a$ is a member of $C$. Thus, we have:

$$
\begin{gathered}
\neg(\operatorname{Has}(a, 1 m) \rightarrow \square \operatorname{Has}(a, 1 m)) \\
a \in C \rightarrow \square a \in C
\end{gathered}
$$

Magnitudes and their corresponding equivalence classes have different modal profiles. This precludes any reduction of the former to the latter. Our analysis of magnitudes needs an intensional element and cannot be given in fully extensional terms.

Does the argument succeed? Although it has substantial force, it raises some interesting questions. First, are all magnitudes had only contingently? If not, then CP's argument has restricted scope: magnitudes that are had by necessity escape its clutches. And in fact, the answer is negative. Consider the cardinality of a plurality of objects, which satisfies the axioms of Suppes' theory and thus qualifies as an extensive magnitude. ${ }^{5}$ But the cardinality of some objects is essential to these objects: some objects could not be those very objects unless they had that cardinality.

Where does this leave us? CP's argument certainly gives the realist a foot in the door. Perhaps this is enough. Once some magnitudes are accepted, why be squeamish about accepting more?

Second, how should we understand transworld comparisons of magnitudes? It seems straightforward that a particular rod might have been $10 \%$ longer than it is. But could all physical objects have been $10 \%$ longer in all directions? For familiar Leibnizian reasons, it is tempting to deny that this scenario is genuinely different from the way things actually are. But if so, what sense can be made of transworld comparisons of length and other magnitudes? I will return to this question shortly.

\section{The representation theorem and its significance}

The second philosophically important theorem of Suppes' theory is a useful representation theorem, which explains why extensive quantities can be measured by positive real numbers.

\footnotetext{
${ }^{4}$ On the appeal to plurals, see Boolos (1984).

5 On CP's analysis, numbers are ascribed, in the first instance, to concepts, not pluralities. This seems the wrong way round. To be $n$ is intrinsic to $x x$, while it is only extrinsic to $F$, namely in virtue of $x x$ being all and only the $F$ 's and $x x$ being $n$ in number, for some $x x$.
} 
Theorem 1 (Representation theorem) Suppose $\mathrm{E}=\mathrm{D}, \preceq, \oplus$ satisfy the theory. Then there is a homomorphism $\mathrm{f}: \mathrm{E} \rightarrow\left\langle\mathbb{R}^{+}, \leq,+\right\rangle$; that is, for all $x$ and $y$ :

$$
\begin{aligned}
& x \leq y \leftrightarrow f(x) \leq f(y) \\
& f(x \oplus y)=f(x)+f(y)
\end{aligned}
$$

Moreover, the homomorphism $f$ is unique up to multiplicative constant.

Underneath this somewhat abstract statement lies an important and easily understood lesson. Extensive quantities can be measured by means of positive real numbers. All we need to do is choose some quantity as a unit, whose measure is therefore 1. Relative to this unit, there is a unique way to assign a measure to every other quantity of this magnitude type.

The theorem is important for several reasons. First, since our only choice is that of a unit, it means that ratios of two magnitudes of one and the same type are absolute. For example, being twice as long, or massive, as some other object is absolute. This absoluteness of ratios plays an important role in the book, both in some of CP's reflections on our representation of magnitudes and in his metaphysical account of the positive real numbers. ${ }^{6}$

Second, the theorem reveals the limited scope of this particular analysis. Only positive reals figure as measurements on this particular analysis, not zero or negative reals. Thus, this analysis excludes magnitudes such as electric charge, which can have both positive and negative values relative to some unit. Nor is any provision made for the complex numbers, infinitesimals, or angular measure in the interval $[0,2 \pi)^{7}$ So the particular analysis just outlined is only a beginning.

Finally, the theorem pinpoints what is required to make sense of transworld comparisons of magnitudes, namely to correlate the units chosen in each of the two possible worlds. Does one of these arbitrarily chosen units represent the same magnitude as the other one or merely some ratio of it? The Leibnizian challenge is that there is no objective answer to this question. Although the challenge is of profound theoretical importance, in practice its force can be blunted. In most ordinary modal theorizing, there is a unique salient correlation. Consider a world in which my meter stick is $10 \%$ larger relative to every other object, but where every other ratio of spatial size remains unchanged. (This characterization is meaningful because ratios are absolute.) Then it is far more natural to choose a unit, in each of the two worlds, among the objects that undergo no relative change, say, someone else's meter stick.

I conclude that the Leibnizian challenge can be met, and that CP's antinominalist argument therefore succeeds.

\footnotetext{
${ }^{6}$ Because of modal considerations, it is important that this account be based on ratios of magnitudes, not of quantities.

${ }^{7}$ This contradicts a claim about the complex numbers on p. 238 (though nothing important hangs on this).
} 


\section{Aristotelian realism and the question of reification}

As adumbrated, CP rejects not only nominalism about magnitudes but also Platonism, at least in the traditional sense mentioned above. ${ }^{8}$ Magnitudes are not transcendent entities, as Plato would have it, but enter into causal and scientific explanations and figure in causal explanatory laws.

CP's Aristotelianism is particularly clear in connection with a novel account of numbers, which are closely related to magnitudes. The central idea of the accountcalled "applicationist individuationism"- is that numbers are individuated in terms of their application conditions. For example:

What makes something the number 1 is that it is the number $n$ such that for an arbitrary concept $F$, for there to be precisely $n F$ s is for [it to be the case that $\exists ! x F x]$ (p. 210)

More generally, let $\exists_{n} x F(x)$ be the first-order formalization of the claim that there are precisely $n F^{\prime}$ s. This is a magnitude property of concepts. Then $n$ is individuated as the number such that, for there to be precisely $n F \mathrm{~s}$ is for it to be the case that $\exists_{n} x F x$. The view that numbers and numerical properties are individuated in terms of their application conditions is distinctly Aristotelian. An obvious advantage of this view is that it removes the sense of mystery about how numbers can be relevant to our study of the physical world. ${ }^{9}$

What about Aristotle's stringent requirement that a property be instantiated in order to exist? Since the natural numbers are understood as cardinality properties of concepts, this requirement threatens to saddle arithmetic with a commitment to an actual infinity of nonmathematical objects. But as $\mathrm{CP}$ observes, it is "quite implausible" that arithmetic should be committed to "infinity in the non-abstract world" (p. 217).

Thankfully, this unpalatable commitment can be avoided. Aristotle himself suggests one option, namely to regard the sequence of natural numbers as merely potentially infinite. Suppose the non-abstract world contains some finite number $M$ of objects. Then only the numbers 0 through $M$ actually exist. But potentially there are more numbers. For necessarily, given any number $N$, possibly there exists a successor $N+1$. This Aristotelian conception of the natural numbers can be proven to succeed in the precise sense that it allows us to interpret all of first-order Dedekind-Peano arithmetic. ${ }^{10}$

CP's preferred way to avoid the unpalatable commitment is to adopt a more relaxed form of Aristotelianism which individuates properties in terms of their possible instances, not only their actual ones. We can thus allow two uninstantiated cardinality properties to be distinct, provided that the two properties differ with regard to their possible instances. The corresponding reified numbers will thus be

\footnotetext{
${ }^{8} \mathrm{He}$ is, however, a Platonist about magnitudes in the widespread contemporary but rather toothless sense of being a realist about these entities.

9 Contrast Field (1989, pp. 18ff) and Kitcher (1978) (whose worries might, however, have force against a truly Platonistic view).

${ }^{10}$ See Linnebo and Shapiro (2019).
} 
individuated as distinct objects via the Reification Link. ${ }^{11}$ Further options exist as well, including one described in Sect. 7, which yields an actual infinity of numbers without relaxing the requirement that properties be instantiated.

I turn now to a different aspect of CP's realism, namely his acceptance of reified magnitudes and numbers. His reasons for this acceptance are much like Frege's (see p. 42). Natural language contains singular terms that refer to magnitudes and numbers. This is also borne out in mathematical practice, which regards numbers and other magnitudes as objects, e.g. by allowing them to be counted and to figure as elements of sets.

Is this reification of magnitudes and numbers defensible? The answer will depend on our ability to answer some more specific questions, which will occupy us in the remainder of this note.

\section{The metaphysical question}

What are reified magnitudes and numbers? In particular, to what extent does the Reification Link shed light on the nature of the object $m$ in terms of the corresponding property $P_{m}$ ?

\section{The question of permissible reification}

Can every magnitude property be reified? Should we adopt a comprehension axiom stating that for every extensive magnitude property $P_{m}$ there is a magnitude proper, $m$, such that the Reification Link holds? Analogous questions arise for numbers.

\section{The metasemantic question}

What is it to refer to a reified magnitude $m$, as opposed to using a predicate with the corresponding magnitude property $P_{m}$ as its semantic value? Analogous questions arise for numbers.

As will transpire, I find CP's answers to the first two questions very congenial and indeed broadly similar to views I have recently defended in Linnebo (2018). We appear to differ, however, concerning the final question.

\section{Shallow nature}

CP claims that the Reification Link provides a complete account of a magnitude in terms of the corresponding magnitude property. The link individuates the magnitude $m$ in terms of corresponding property $P_{m}$, that is, it provides an account of "what makes $m$ the object it is". The magnitude $m$ has, as I will put it, a shallow nature

\footnotetext{
11 A closely related option, suggested by $\mathrm{CP}$, is to retain the requirement that the cardinality properties to be instantiated but to insist that the reified numbers exist necessarily. From a purely technical point of view, this option isn't very different from CP's preferred one.
} 
relative to the property $P_{m} \cdot{ }^{1213}$ Again, CP's view becomes particularly clear in the case of numbers, which are said to have no nature beyond what is contained in their individuation: "there is nothing more to being any given number than is given in the individuating condition" (p. 141). More generally, "the very nature of abstract objects is explained by their application conditions."

The idea of shallow nature applies not only to numbers and other abstract objects but also to relations between them. Consider the successor relation $S$ that holds between any natural number and its immediate successor. $S$ holds between two numbers $m$ and $n$ just in case $n$ applies to concepts with precisely one more instance than concepts to which $m$ applies.

This is in effect just Frege's famous characterization of the successor relation: ${ }^{14}$

$$
\text { Smn } \leftrightarrow \exists \exists a(F a \wedge m=\# x .(F x \wedge x \neq a) \wedge n=\# x . F x)
$$

Again, CP makes a claim about shallow nature: "There is no more for two natural numbers to stand in the successor-of relation than the displayed condition's holding" (p. 215).

The result is an attractive and broadly neo-Fregean metaphysics of the abstract. While there are numbers and other abstract objects, there is nothing more to these objects and the relations in which they stand than what is contained in certain corresponding magnitude properties.

\section{Asymmetric abstraction}

The question of permissible reification requires a bit of background. Reification is dangerous, as Frege painfully discovered. His claim that every property $F$ can be reified as a corresponding extension $\hat{u . F u}$ was famously refuted by Russell's paradox. The problem concerns Frege's Basic Law V, which we can formulate as follows in order to highlight the structure it shares with the Reification Link:

$$
\operatorname{Has}(x, \hat{u} . F u) \leftrightarrow F x
$$

Of course, extensions of concepts aren't magnitudes or numbers. But $\mathrm{CP}$ recognizes that there is a notion of ordinality, which is measured by an ordinal number. And the Reification Link for ordinality gives rise to the Burali-Forti paradox (that is, the

\footnotetext{
12 For some closely related ideas, see Hale and Wright (2007) and Linnebo (2018, §11.3).

13 This view plays a crucial role in CP's response to the notorious Julius Caesar problem.

A natural number is individuated by its application conditions, in numerical quantifications. Any object that is not individuated by its application conditions in numerical quantifications is not a natural number. Julius Caesar is not so individuated. So no natural number is identical with Julius Caesar. (p. 214)
}

This response is closely related to the neo-Fregeans' (Hale and Wright, 2001b), which seeks to distinguish Caesar from any natural number on the grounds that the two objects are subject to different criteria of identity.

14 Instead of this characterization, CP gives a more complicated, but ultimately equivalent characterization. 
paradox of the ordinal of the well-ordering of all ordinals). ${ }^{15}$ Thus, even the reification of numbers is dangerous unless constrained in some way.

Simultaneously, the constraints imposed must not be too severe, as CP clearly recognizes. In fact, although his approach to philosophy differs markedly from Carnap's, CP goes out of his way to commend Carnap's liberal and permissive attitude towards the existence of mathematical objects.

Is there a way to balance these two conflicting pressures-for safety against paradox, on the one hand, and a liberal approach to mathematical ontology, on the other? This has become known as the bad company problem. We want to excise all "bad companions", such as Basic Law V and the problematic form of ordinal abstraction, while retaining all "good" cases of abstraction or reification. Ideally, the line of demarcation should also be well motivated and suitably integrated with our philosophical account of abstract objects. This is obviously a tall order.

Although CP, like most commentators, doesn't provide a worked-out answer to the bad company problem, he makes some tantalizing suggestions, which point in the same direction as an attempted solution recently proposed by myself (Linnebo 2018, ch. 3). Consider the celebrated Hume's Principle, which describes how cardinal numbers are obtained by abstraction on concepts:

$$
\# F=\# G \leftrightarrow F \approx G
$$

A question of great philosophical importance arises. What is the relation between the two sides of this abstraction principle? The prevailing neo-Fregean view has been that the two sides are symmetrically related: these are just two different ways to "carve up" one and the same fact. ${ }^{16}$ In Linnebo (2012), which CP quotes with approval, I complained that on this symmetrical conception any problematic features attaching to one side would be inherited by the other side. Instead, both of us emphasize certain asymmetric features of (HP) and other acceptable abstraction principles, namely that matching instances of the two sides differ with respect to (i) which objects they refer to; (ii) their ontological commitment; and (iii) metaphysical explanation (which flows right-to-left, not left-to-right).

The resulting asymmetric conception of abstraction holds great promise with respect to the bad company problem. It suggests, as CP puts it, a "hierarchy of individuation" (p. 228). Abstract objects are individuated successively, starting with material made available by the physical world, perhaps including its modal aspects. Once a certain stock of abstract objects have been individuated, they can be used to individuate yet further abstract objects. Paradox is avoided by ensuring that, throughout this stepwise individuation, we only ever draw on objects and truths that are available at that stage. For example, we must require that

in the individuation of any particular natural number, the individuating condition not involve quantification over, or involve reference to, that very number whose individuation is in question. (p. 224)

\footnotetext{
15 See Hodes (1984, p. 138).

16 See Hale and Wright (2001a) and Rayo (2013), as well as Linnebo (2018, Sect. 1.7 and ch. 4) for discussion and further references.
} 
More generally, the asymmetric conception of abstraction suggests a grounded, or broadly predicative, approach to abstraction, where at any stage we can only appeal to entities and truths available at that stage. The details are subtle but need not detain us here. ${ }^{17}$

Notice that the asymmetric conception of abstraction permits Fregean "bootstrapping". Suppose we have established the existence of the numbers $0,1, \ldots, N$. Since numbers are bona fide objects, they can figure in further instances of abstraction. This enables us to establish the existence of $N+1$ by cardinality abstraction applied to the mentioned list. We now repeat the argument, only this time starting with the longer list of numbers $0,1, \ldots, N+1$. By iterating further, we establish the existence of infinitely many numbers-without any unpalatable assumptions about the cardinality of the non-abstract world and (if desired) without lifting the Aristotelian requirement that properties be instantiated.

\section{The representation of magnitudes and numbers}

The book develops a rich and detailed account of how animals (including humans) represent magnitudes. The central idea is that magnitudes external to the mind are represented by magnitudes in the mind or brain. This account nicely explains how perception of magnitudes is unit-free, how we sometimes represent ratios of magnitudes, and how analogue computation works (namely by law-governed operations on the representing magnitudes which generate further representing magnitudes).

I have little to add here, other than to observe that the account is most plausibly understood as an account of the representation of magnitude properties, not as an account of singular reference to reified magnitudes. Indeed, much of the attraction of the account is that it applies also to simpler animals (such as birds) and to largely innate cognitive capacities (what (Carey 2009) calls "core cognition").

What is it, then, for a thinker to explicitly represent a number or some other abstract object? Some philosophers have argued that such representation is impossible, on the grounds that representation is always based on some causal interaction with the object represented, which is impossible when that object is abstract. CP dismisses these concerns and proposes an entirely non-causal explanation of how a thinker represents an abstract object. "In cases where the principle 'Individuation Precedes Representation' holds good", he writes, the representation of an abstract object "involves only drawing in the right way on the metaphysics of the entities in question" (p. 209).

To put some flesh on the bone, let us consider a representative example of this explanatory strategy:

to think of a natural number as 1 , for instance, is to have tacit knowledge that for there to be $1 F$ is for it to be the case that there to something that is $F$, and nothing else is. (p. 229)

17 The interested reader may consult (Linnebo 2018, ch. 3). 
Notice that, whereas the explanandum involves a cardinal number, the explanans involves only a cardinality property.

This may strike you as metasemantic alchemy. How can tacit knowledge of a cardinality property be transmuted into an explicit representation of the corresponding cardinal number, which is an abstract object? CP's response appeals to the shallow nature of the object vis-à-vis the property.

This is an account in which Individuation Precedes Representation, [...] because the condition for thinking of a natural number $n$ mentions the condition, constitutive of $n$, for there being $n F$ s. If there were more to being $n$ than that relation to numerical quantification holding, no doubt we would require more for thinking of or representing $n$. (pp. 230-231)

The appeal to shallow nature shows that a response to the alchemy charge may well be possible. But by itself, this hardly constitutes a worked-out response. As Frege himself points out, knowledge of a cardinality property does not by itself suffice for representation of the corresponding cardinal number. ${ }^{18}$

How can we bridge the gap between tacit knowledge of a cardinality property and explicit representation of the corresponding cardinal number? Frege's brilliant proposal from the famous $\$ 62$ of his Foundations of Arithmetic is to base the representation of a cardinal number-and of other objects as well-on criteria of identity. To represent an object, Frege argues, it suffices to possess a criterion of identity for the would-be referent, where this criterion doesn't itself presuppose the very relation of reference we are trying to explain. Admittedly, Frege's proposal too is fairly programmatic, and much philosophical work remains. The neo-Fregeans have had a go at this, and so have I. ${ }^{19}$ My parting question to $\mathrm{CP}$ is whether he too intends to appeal to criteria of identity to account for the constitution of reference to numbers and other abstract objects, and if not, how else he intends to bridge the mentioned gap-shallow though it may be.

Funding Open Access funding provided by University of Oslo (incl Oslo University Hospital)

Open Access This article is licensed under a Creative Commons Attribution 4.0 International License, which permits use, sharing, adaptation, distribution and reproduction in any medium or format, as long as you give appropriate credit to the original author(s) and the source, provide a link to the Creative Commons licence, and indicate if changes were made. The images or other third party material in this article are included in the article's Creative Commons licence, unless indicated otherwise in a credit line to the material. If material is not included in the article's Creative Commons licence and your intended use is not permitted by statutory regulation or exceeds the permitted use, you will need to obtain permission directly from the copyright holder. To view a copy of this licence, visit http:// creativecommons.org/licenses/by/4.0/.

\footnotetext{
18 See Frege (1953, §§56-57).

19 See Wright (1983) and Hale and Wright (2001a) and Linnebo (2018) (esp. chs. 2 and 8), respectively.
} 


\section{References}

Boolos, G. (1984). To be is to be a value of a variable (or to be some values of some variables). Journal of Philosophy, 81(8), 430-449.

Carey, S. (2009). The origin of concepts. Oxford: Oxford University Press.

Field, H. (1989). Realism, mathematics, and modality. Oxford: Blackwell.

Frege, G. (1953). Foundations of Arithmetic (J. L. Austin, Trans.). Blackwell, Oxford.

Hale, B., \& Wright, C. (2001a). Reason's proper study. Oxford: Clarendon.

Hale, B., \& Wright, C. (2001b). To bury Caesar.... In (Hale and Wright, 2001a).

Hale, B., \& Wright, C. (2007). Abstraction and additional nature. Philosophia Mathematica, 16(2), 182-208.

Hodes, H. (1984). Logicism and the ontological commitments of arithmetic. Journal of Philosophy, 81(3), 123-149.

Hossack, K. (2020). Knowledge and the philosophy of number: What numbers are and how they are known. London: Bloomsbury.

Kitcher, P. (1978). The plight of the platonist. Noûs, 12, 119-136.

Linnebo, Ø. (2012). Metaontological minimalism. Philosophy Compass, 7(2), 139-151.

Linnebo, Ø. (2018). Thin objects: An abstractionist account. Oxford: Oxford University Press.

Linnebo, Ø., \& Shapiro, S. (2019). Actual and potential infinity. Noûs, 53(1), 160-191.

Peacocke, C. (2015). Magnitudes: Metaphysics, explanation, and perception. In Coliva, A., Munz, V., \& Moyal-Sharrock, D. (Eds.), Mind, language and action: Proceedings of the 36th international Wittgenstein symposium (pp. 357-388). De Gruyter.

Peacocke, C. (2019). The primacy of metaphysics. Oxford: Oxford University Press.

Putnam, H. (1969). On properties. In R. Nicholas (Ed.), Essays in Honor of Carl G. Hempel: A tribute on the occasion of his sixty-fifth birthday (pp. 235-254). Dordrecht: Springer. Reprinted in (Putnam, 1975).

Putnam, H. (1975). Mathematics, matter and method. Cambridge: Cambridge University Press.

Rayo, A. (2013). The construction of logical space. Oxford: Oxford University Press.

Suppes, P. (1951). A set of independent axioms for extensive quantities. Portugaliae Mathematica, 10(4), $163-172$.

Wright, C. (1983). Frege's conception of numbers as objects. Aberdeen: Aberdeen University Press.

Publisher's Note Springer Nature remains neutral with regard to jurisdictional claims in published maps and institutional affiliations. 